\title{
Review
}

\section{Research Progress on Systemic Lupus Erythematosus Complicated with Infection}

\author{
Weisan Zhang \\ Department of Geriatrics, Tianjin Medical University General Hospital, Tianjin, China
}

\section{Keywords \\ Systemic lupus erythematosus; Infection; \\ Antinuclear; Epstein-Barr virus}

\section{Correspondence}

Weisan Zhang,

E-mail: wszhangmy@163.com

DOI: $10.1515 / i i-2017-0106$

\begin{abstract}
In recent years, in treatment standardization of systemic lupus erythematosus (SLE), infections and serious complications became the leading cause of death related to this disease, exceeding those of renal involvement and lupus encephalopathy. SLE coinfection is mainly related to defects in humoral immunity and cellular immunity, SLE disease activity, and doses of hormone and immune inhibitors.
\end{abstract}

Systemic lupus erythematosus (SLE) is a connective tissue disease, which is mediated by autoimmunity with characteristic symptoms of immunity inflammation. Two major clinical features of SLE are as follows: a variety of autoantibodies and multisystem involvement with antinuclear antibody (ANA) as representative in serum. Cause of the disease remains unknown. To date, SLE is considered an autoimmune disease mainly caused by heredity, environment, and infection. With further understanding of pathogenesis of SLE and improvement of diagnosis and treatment, prognosis of SLE and 10-year survival rate significantly improved. Infection displays a noticeable relationship with occurrence, development, and prognosis of SLE; it is a primary cause of progression and death associated with SLE ${ }^{[1]}$. Recently, with treatment standardization of SLE, resulting infection and serious complications became the leading causes of death in SLE patients, exceeding those of renal involvement and lupus encephalopathy ${ }^{[1]}$. Some studies pointed out that more severe infections occurred in disease progress of $1 / 3-1 / 2$ SLE patients ${ }^{[2]}$. Incidence of coinfection in SLE patients is approximately 10 times higher than that in patients with rheumatoid arthritis and approximately five times higher than that in patients with cancer ${ }^{[3]}$. Infection is mainly related to defects in humoral immunity and cellular immunity, SLE disease activity, and dose of hormone and immune inhibitors. These components represent independent risk factors for SLE patients complicated with infection.

\section{Research progress on different pathogen infections}

\section{Epstein-Barr virus (EBV)}

EBV infection, which aggravates SLE, became a significant research topic in the past 30 years locally and abroad. Pathogenic mechanism of this infection may be the abnormal activation of B lymphocytes, which are activated by EBV, and $\mathrm{T}$ cell immune clearance dysfunction. Evidence showed that $B$ cells infected by EBV in SLE patients are 10 times higher than those in healthy population ${ }^{[4]}$. High homology was observed between EBV multiple antibodies and SLE in vivo autoantibodies ${ }^{[5-7]}$. Activated EBV present specific BamHI-w gene segment ${ }^{[8]}$, with high-level specific antigens EBV nuclear antigen 2, BZLF1, and latent membrane protein 1 (LMP-1) ${ }^{[9]}$, activated CD21 and CD23, and highly expressed LMP1 and apoptosis-related gene bcl-2. Dendritic cells (DC) participate in innate immune response mechanism in SLE through interferon $\alpha$ (IFN- $\alpha$ ), which is induced by TLR4 or TLR9 signal pathway ${ }^{[10]}$. After reactivation of the virus, EBV specific $\mathrm{CD} 4{ }^{+} \mathrm{CD} 69^{+}$cells highly express tumor necrosis factor or reduce ability of IFN- $\alpha$ molecules and CD8+ cells to secrete cytokines ${ }^{[1,12]}$. These findings suggest that EBV infection is closely related to onset and exacerbation of SLE. No symptom exists during the first EB virus infection in childhood. During first infection in 
puberty, probability of infectious mononucleosis (IM) reaches $30 \%-70 \%$, and approximately $20 \%$ of B cells in vivo are involved at that period ${ }^{[13]}$. EB infection rate totals $99.6 \%$ in all children with SLE ${ }^{[13]}$. IM and SLE exhibit similar clinical manifestations and also express a variety of ANAs. Several cases were observed from IM to development of SLE. These epidemiological data clearly showed correlation of these conditions. However, some studies revealed that high expressions of EBV antibody and DNA in SLE patients do not affect total activity change in SLE ${ }^{[14]}$.

\section{Fungus}

With wide applications of glucocorticoids, immunosuppressants, and broad-spectrum antimicrobial agents, fungal infection became one of the important causes of death in critically ill patients. Mortality rate caused by fungal infection leads that of SLE coinfection ${ }^{[12,15]}$. Mortality rate of SLE infected with Aspergillus reaches $80 \%{ }^{[16]}$. Therefore, prevention and treatment of fungal infection can improve survival rate of SLE patients. Difficulty arises from distinguishing manifestations of fungal infection from those of primary diseases or bacterial infection because of mutual doping. Diagnosis depends on etiological examinations. A multivariate analysis results showed that primary lupus lung disease, combined application of two kinds and mentioned antibacterial drugs, daily dose of glucocorticoid, and using antibiotics for more than two weeks are risk factors for secondary fungal infection ${ }^{[17]}$. All fungal infection patients should be treated with active antifungal agents. According to guidelines for treatment of pulmonary fungal infections issued by the American Thoracic Society in $2011^{[18]}$, single or combined application of fluconazol, amphotericin $B$, itraconazole, voriconazole, and other drugs must be administered to control fungal infection.

\section{Bacterium}

Bacterial infection is the most common in SLE coinfection, accounting for $49 \%-80 \%{ }^{[19]}$. Among $\mathrm{G}^{+}$bacteria, Staphylococcus aureus and Streptococcus are the most common causes of infection ${ }^{[20]}$. In $\mathrm{G}^{-}$bacteria, Escherichia coli, Klebsiella, and Pseudomonas aeruginosa primarily results in infection. Serious and fatal bacteremia and septicemia are often caused by these bacteria ${ }^{[21]}$. To date, foreign studies showed that independent risk factors of SLE complicated with bacterial infection include disease activity, complement level, $24 \mathrm{~h}$ urinary protein quantity, immune inhibitors, and hormone use ${ }^{[22]}$. Domestic materials proved that independent risk factors of SLE coinfection comprise C3, 24 h urinary protein quantity, cyclophosphamide cumulant, daily dose of glucocorticoid, and excessive and frequent use of broad-spectrum antibiotics ${ }^{[23]}$. Respiratory infection is the most common infection in SLE complicated with bacterial infection ${ }^{[24]}$, followed by urinary tract infection and gastrointestinal infection.

\section{Tuberculosis (TB)}

SLE complicated with TB infection presents early atypical symptoms. Hormones and immunosuppressants can inhibit cellular immunity and reduce inflammatory reaction, inhibiting and masking symptoms of TB. Patients manifest fever, sweating, fatigue, and other nonspecific manifestations in clinical settings; these symptoms are often mistakenly regarded as SLE disease activities. Clinically, SLE complicated with TB is easily mistaken as pulmonary manifestation. TB complicated by SLE includes infiltrative pulmonary TB and miliary TB. Pulmonary lesions are constantly related to low immunity of SLE patients. Extrapulmonary TB (20\%-52\%) easily occurs, ${ }^{[25]}$ and it involves bones, joints, cerebrospinal fluid, kidneys, soft tissues, and blood-borne factors and differs from pulmonary TB of ordinary population. Lunch et al. ${ }^{[26]}$ reported that when transplantation patients are complicated with $\mathrm{TB}$, tuberculin skin test (PPD) shows $70 \%$ negative results. Positive results are not easily obtained in PPD test because of the use of hormones and immunosuppressants. Therefore, PPD test features limited diagnostic value to SLE-complicated TB. One suggested method is performing repeated detection by acid-fast bacilli in sputum smear for SLE patients with suspected TB. For patients with negative TB, TB antibody and T-SPOT test should be performed to indirectly diagnose TB infection. Populations who use immunosuppressant for long periods, with history of TB, hypoproteinemia, and less $\mathrm{T}$ cell subgroup $\mathrm{CD} 4$ cells, are easily complicated with $\mathrm{TB}$ infection. Kim et al. ${ }^{[27]}$ investigated 269 cases of rheumatism complicated with TB. Results showed significant relation of daily mean dose and accumulative quantity of glucocorticoid and hormone shock therapy to TB incidence. Sayarlioglu et al. ${ }^{[28]}$ studied and showed that when daily mean dose and total dose of glucocorticoid for SLE patients are high and with long administrative time, probability of $\mathrm{TB}$ occurrence is high. Isoniazid, rifampicin, and p-aminosalicylic acid can induce lupus-like syndrome. Studies revealed that approximately $20 \%$ of patients taking isoniazid orally can yield positive 
ANAs. Only less than $1 \%$ of patients with clinical symptoms should cease drug administration. After taking medications, clinical manifestations include fever, butterfly erythema, lymph node enlargement, and dropsy of serous cavity. Laboratory inspection can detect anemia, leukopenia, ANA positivity, and antihistone antibodies. Additional research should distinguish whether these symptoms are caused by lupus activity, recrudescence, or drugs administered. Studies should also verify whether SLE patients receiving long-term glucocorticoid treatment use isoniazid to prevent TB. Some studies showed that oral intake of isoniazid can decrease incidence of SLE complicated with TB from $11 \%$ to $2 \%{ }^{[30]}$. Some studies also indicated unclear prevention effect of isoniazid; using it in non-endemic areas is unnecessary ${ }^{[31]}$.

\section{Pneumocystosis (PCP)}

In recent years, many SLE cases complicated with reported Pneumocystis carinii pneumonia (PCP) infection were observed. Showing rapid progress and high mortality, SLE complicated with PCP exhibits no characteristic clinical manifestation but mainly presents dry cough, fever, progressive dyspnea, type I respiratory failure, and hypoxemia. Imaging mainly showed pulmonary interstitial changes. Difficulty arises from distinguishing lupus pneumonia, fungal pneumonia, and cytomegaloviral pneumonia. This condition indicates that when fever cannot be easily corrected, and dyspnea occurs in SLE-diseased children using glucocorticoids and immunosuppressant with no visible symptoms, chest imaging should be performed in a timely manner. Clinical consideration should be provided to combination of PCP. PCP is an infectious disease, which can be cured early but easily results in misdiagnosis and missed diagnosis. Early treatment is key to addressing these issues. To date, some literature reported that daily dose of glucocorticoid $\geq 16 \mathrm{mg}$ and treatment of more than eight weeks are high-risk factors for PCP. Some scholars suggested that when daily dose of glucocorticoid $\geq 20 \mathrm{mg}$, and treatment process is more than one month, PCP prophylaxis may occur ${ }^{[32]}$. Some foreign scholars believed that another risk factor for PCP includes absolute values of CD4 positive T cells among high-risk populations who use glucocorticoids and immunosuppressant alone or in combination $<0.3 \times 10^{9} / \mathrm{L}$ ${ }^{[33]}$. Thus, preventive treatment should be administered. Therefore, by regularly conducting $\mathrm{T}$ cell subgroup test to SLE-diseased children, determining PCP high-risk patients becomes possible. Some benefits may be observed in early prevention ${ }^{[33]}$.

\section{Diagnostic evaluation index}

\section{Procalcitonin (PCT)}

After nearly 20 years of research and practice, PCT was recommended as an evaluation indicator for diagnosis, stratification, treatment monitoring, and prognosis of bacterial infection sepsis ${ }^{[3,34]}$. However, internationally, controversies exist regarding diagnostic evaluation value of PCT in SLE and other autoimmune diseases complicated with infection or sepsis. Some studies revealed high correlation of severity of SLE infection with PCT concentration. PCT value in $\mathrm{G}^{-}$bacterial infection is significantly higher than that in $\mathrm{G}^{+}$bacterial infection. Escherichia coli and Klebsiella pneumoniae infection are the most significant $\mathrm{G}^{-}$bacterial infection. Sustained increase in PCT indicates sustained inflammatory response. Infection is controlled after application of antibiotics, and PCT value decreases. However, in SLE complicated with $\mathrm{G}^{+}$bacterial infection, increase in PCT does not parallel severity of disease ${ }^{[35,36]}$.

\section{C response protein (CRP)}

Some researchers observed that differences exist in CRP concentrations between SLE activity group, systemic bacterial infection group, local bacterial infection group, and control group. Through comparison, differences were also noted between systemic bacterial infection group, SLE activity group, and local bacterial infection group. No visible difference was observed between SLE activity group and local bacterial infection group. These findings indicate that increased CRP presents clinical value in identifying SLE activity and SLE complicated with bacterial infection ${ }^{[33]}$. However, CRP also increases significantly in complicated fungal infection and serositis. Specificity of CRP is low similar to that of diagnosing SLE bacterial infection ${ }^{[33]}$.

\section{CD64 index}

Flow cytometry was used to detect mean flourscence intensity of CD64 on surfaces of peripheral blood neutrophil granulocytes, monocytes, and lymphocytes to calculate CD64 index. The aim was to identify SLE secondary infection and disease activity. Some studies revealed that CD64 index bears significantly positive correlation with white blood cell count, number of neutrophils, erythrocyte sedimentation rate, CRP, and PCT. CD64 index showed no significant correlation with SLE disease activity index, ANA, 
anti-ds-DNA antibody, and C3 and C4 levels. These findings indicate that CD64 index can be used as marker for SLE secondary bacterial infection and disease activity ${ }^{[37]}$.

\section{ImmuKnow immune cell function assay}

ImmuKnow immune cell function assay is used to detect contents of adenosine triphosphate (ATP) released from $\mathrm{CD}^{+} \mathrm{T}$ lymphocytes in peripheral blood of patients after stimulation. This test aims to determine immune responses mediated by organism cells. Research showed that ATP contents in $\mathrm{CD} 4^{+} \mathrm{T}$ lymphocytes correlate well with immune state of organisms after organ transplant. This finding can be used as reference index for monitoring immune status of patients ${ }^{[38]}$. Some research results indicated that ATP value of $\mathrm{CD}^{+} \mathrm{T}$ lymphocytes of healthy Chinese lies in middle reaction zone. In lupus renal infection, ATP value is significantly lower than those of non-infection and health control groups. Low ATP content of $\mathrm{CD}^{+}{ }^{+} \mathrm{T}$ lymphocytes is an independent risk factor for lymph node secondary infection. This result implies that ATP value of $\mathrm{CD} 4^{+} \mathrm{T}$ lymphocyte is a strong index for assessing risk of infection not only in organ transplant patients but also in lupus renal infection individuals ${ }^{[38,39]}$. These findings imply that ImmuKnow assay can be used to monitor immune function state of lupus renal infection patients and predict their infection risk.

\section{Application of vaccines}

Vaccine application remains controversial. According to previous assumptions, some vaccines may induce SLE activity. However, to date, clinical data show that inactivated vaccines, especially Streptococcus pneumonia and influenza virus vaccine, are safe and effective for patients with SLE. For SLE patients using immunosuppressant and prednisone (>20 mg/day), use of inactivated vaccines is discouraged. Immunosuppressants should be discontinued three months before vaccination, and their use can be continued two to four weeks after injection.

\section{Declarations}

\section{Acknowledgements}

No.

\section{Competing interests}

The author declare that he has no competing interest.

\section{Authors' contributions}

WS Zhang made the literature analysis and wrote, discussed and revised the manuscript of this review.

\section{References}

1 Fei Y, Gan F, Hou Y. Causes of death of systemic lupus erythematosus patients in the past twenty-five years. Chinese Journal of Rheumatology, 2012,16(9):596-600.

2 Mok M, Wong S, Chan T, et al. Non-tuberculous mycobacterial infection in patients with systemic lupus erythematosus. Rheumatology, $2007,46(2): 280$

3 Chen H, Tsai WP, Leu HS, et al. Invasive fungal infection in systemic lupus erythematosus: an analysis of 15 cases and a liter-aturreview. Rheumatology, 2007,46(3):539.

4 Draborg AH, Duus K,Houen G. Epstein-Barrvirus in systemic autoimmune diseases[J]. Clin Dev Immunol,2013,2013:535738.

5 Barzilai O, Sherer Y, Ram M, et al. Epstein-Barr virus and cytomegalovirus in autoimmune diseases:are they truly notorious? Apreliminary report. Ann N Y AcadSci, 2007,1108: 567-577.

6 Mcclain MT, Poole BD, Bruner BF, et al. An altered immune response to Epstein-Barr nuclear antigen 1 in pediatric systemic lupus erythematosus. Arthritis Rheum, 2006, 54:360-368.

7 Harley JB, James JA. Epstein-Barr virus infection induces lupusautoimmunity. Bull NYU HospJt Dis, 2006, 64:45-50.

8 Li Yi. EB Study of the relationship between Epstein-Barr virus infection and abnormal activation of B-lymphocytes in systemic lupus erythematosus. Chinese Journal of Rheumatology, 2009, 13(6):394-396.

9 Wang L, Wang J, Pan L, et al. The study on the possible pathogenesis of EBV latent membrane protein 1 in inducing systemic lupus erythematosus. Chinese Journal of Rheumatology, 2011, 15(10):707-709.

10 Quan TE, Roman RM, Rudenga BJ, et al. Epstein-Barr virus promotes interferon-alpha production by plasmacytoid dendritic cells. Arthritis Rheum, 2010, 62:1693.

11 James JA, Robertson JM. Lupus and Epstein-Barr. Curr Opin Rheumatol, $2012,24: 383-388$.

12 Larsen M, Sauce D,Deback C, et al. Exhausted cytotoxic control of Epstein-Barr virus in human lupus. PLoS Pathog, 2011, 7:e1002328.

13 Tattevin P, Le Tulzo Y, Minjolle S, et al. Increasing incidence of severe Epstein-Barr virus-related infectious mononucleosis: surveillance study. J Clin Microbiol, 2006, 44(5): 1873-1874.

14 Zhang X, Feng X. Relevance of the Epstein Barr virus infection with systemic lupus erythematosus. Chinese Journal of clinicians, 2013, 7(14):6349-6354.

15 Mai C, Zhang D, Ye R. Analysis of causes of death in 90 patients with systemic lupus erythematosus. Chinese Journal of Nephrology, 1998, 14(2):114-116.

$16 \mathrm{Hu} \mathrm{J}$, Li D. Analysis of factors associated with death in patient with 
systemic lupus erythrmatosus . Southern China Journal of DermatoVenereology, 2004, 11(4):325-326. infection. Beijing: Chinese Peking Union Medical College, 2006.

18 Jimper AH, Knox Ks. An official American Thorack Society statement: treatment of fungal infections in adult pulmonary and critical care patients. Am J RespirCrit care Med, 2011,183(1):96-128.

19 Szeto CC, Kwan BC, Lai FM, et al. Tacrolimus for the treatment of systemic lupus erythematosus with pure class V nephritis. Rheumatology (Oxford), 2008,47(11):1678-1681.

20 Chen HS, Tsai WP, Leu HS, et al. Invasive fungal infection in systemic lupus erythematosus:an analysis of 15 cases and a literature review. Rheumatology, 2007, 46(3):539-544.

$21 \mathrm{Wu} \mathrm{X}$, Chen $\mathrm{N}$. The clinical characteristics and early diagnosis of systemic lupus erythematosus complicated with infection. Chinese Journal of Practical Internal Medicine, 2010, 30(2):154-156.

$22 \mathrm{Ng} \mathrm{W}$, Chu C, Yuen $\mathrm{K}$, et al. Lymphopenia at presentation is associated with increased risk of infections in patients with systemic lupus erythematosus. QJM, 2006, 99(1):37.

23 Jiang Y, Zhao Y, Wei W. Analysis of clinical characteristics of patients with systemic lupus erythematosus complicated with infection. Journal of Tianjin Medical University, 2010, 16(2):295-297.

24 Juarez M, Misischia R, Alarcon GS. Infections in systemic connective tissue diseases: systemic lupus erythematosus, scleroderma, and polymyositis/dermatomyositis. Rheum Dis Clin North Am, 2003, 29(1):163-184.

25 Li Zhijun, Wang Cuilan, Fan Xiaoyun, et al. Clinic study on systemic lupus erythematosus complicated with bacterial and fugal infection. Chinese Journal of Rheumatology, 2000, 4(3):165-168. in a corticosteroid-treated rheumatic disease patient-population. Clin Exp Rheumatol, 1998, 16(1):9-13 . Sayarlioglu M. Tuberculosis in Turkish patients with systemic-lupus erythematosus:increased frequency of extrapulmonarylocalization.
Lupus, 2004,13(4):274-278.

29 Mok MY, Lo Y, Chan TM,et a1. Tuberculosis in systemic lupus erythematosus in an endemic area and the role of isoniazid prophylaxis during eorticosteroidtherapy. J Rheumatol, 2005, 32:609-615.

30 Falagas ME, Voidonikola PT, Angelousi AG. Tuberculosis in patients with systemic rheumatic or pulmonary diseases treated with glueocorticosteroids and the preventive role ofisoniasid: a review of the available evidence. Int J Antimierob Agents, 2007, 30:477-486.

31 Plakke MJ, Jalota L, Lloyd BJ. Pneumocystis pneumonia in a non-HIV patient on chronic corticosteroid therapy: a question of prophylaxis. BMJ Case Rep, 2013. pii: bcr2012007912. doi:10.1136/bcr-20120007912.

32 Mansharamani NG, Balachandran D, Vemovsky I,et al. Peripheral blood CD4+T-lymphocyte counts during Pneumocystis carinii pneumonia in immanocompromised patients without HIV infection. Chest, 2000,118:712-720.

33 Vogawa J, Harigai M, Nagasaka K, et a1. Prediction of and prophylaxis against Pneumocystis pneumonia in patients with connective tissue diseases undergoing medium-or high dose corticosteroid therapy. Mod Rheumat, 2005,15:91-96.

34 He B, Zhu Y. Application of procalcitonin in children with sepsis. Chinese Pediatric Emergency Medicine, 2013, 20(3):324-326.

35 Yao Dongyun, Huo Heshui. Rapid identification of procalcitonin in systemic lupus erythematosus activity and bacterial infection. Chinese Journal of clinical immunology and allergy, 2014, 221:227.

36 Chang S, Li G, Wu Y, et al. The value of calcitonin and high sensitive $\mathrm{C}$ reactive protein in the diagnosis of neonatal septicemia. Chinese Pediatric Emergency Medicine, 2013, 20(4):411-413.

37 You H, Qiu Y, Wu L, et al. The significance of CD64 index in the identification of systemic lupus erythematosus secondary infection and disease activity. Jiangsu Medicine Journal, 2014, 40(1):53-55.

38 Zeevi A, Lunz J. CylexImmuKnow cell function assay. Methods Mol Biol, 2013, 1034:343-351.

39 Bhorade SM, Janata K, Vigneswaran WT, et al. CylexImmu Know assay levels are lower in lung transplant recipients with infection. J Heart Lung Transplant, 2008, 2008: 990-994. 